\title{
Fruit consumption and risk of type 2 diabetes: results from three prospective longitudinal cohort studies
}

\begin{abstract}
The authors of this Research paper have informed us of a coding error in their analysis programmes that generated the results for this study (BMJ 2013;347:f5001, doi:10.1136/bmj.f5001). Specifically, the total fruit intake variable included both whole fruits and fruit juices in the Nurses' Health Study and Health Professionals Follow-up Study, but the authors incorrectly treated it as whole fruit in their initial analyses. Consequently, some of the values recorded for the hazard ratios and $95 \%$ confidence intervals of type 2 diabetes for whole fruit intake and fruit juice intake are incorrect. The authors state that these erroneous values do not affect the conclusions of their study or their estimates for individual fruits, and would also like to clarify that the total fruit intake variable was correctly coded in the Nurses' Health Study II. The corrections that should be made to this paper as a consequence of the authors' coding mistake are listed below.
\end{abstract}

In both the results section of the abstract and the third paragraph of the long results section, the pooled hazard ratio $(95 \%$ confidence interval) of type 2 diabetes for every three servings/week of total whole fruit consumption should have read 0.98 (0.97 [not "0.96"] to 0.99). This inaccurate lower confidence interval is also listed in table 2 , where it has been corrected in the republished version below. In addition, this error appeared in the print version, which can be seen online in the "Section PDF," under the paragraph "Main results and the role of chance" and in the figure.

In the results section of the abstract, the penultimate sentence should have read that the "pooled hazard ratio for the same increment in fruit juice consumption was 1.07 (1.04 to 1.09), not "1.08 (1.05 to 1.11)." Readers should also note that this incorrect hazard ratio is listed in table 4, where it has been corrected in the republished version below. This inaccurate value for the hazard ratio also appeared in the print version, which can be seen online in the "Section PDF," under "Main results and the role of chance" and in the figure.

Under the methods section, the seventh sentence in the paragraph "Assessment of fruit consumption" should have been truncated to read: "We calculated total whole fruit consumption by summing the consumption levels of the 10 individual fruits."
In the first paragraph of the results section, the last two sentences contain some inaccuracies. The penultimate sentence should have noted that the average rate of loss to follow-up for each two year follow-up cycle was $0.7 \%$ [not " $0.8 \%$ "] for $<4$ servings/week and $0.7 \%$ for $\geq 3$ servings/day of total fruit consumption in the Nurses' Health Study. While the last sentence should have read that these respective values were $0.9 \%$ and $1.3 \%$ [not " $1.0 \%$ and $1.1 \%$ "] in the Health Professionals Follow-up Study.

In the last sentence of the second paragraph in the results section, the Spearman correlation coefficients for total whole fruits in relation to the modified alternate healthy eating index score should have been 0.29 [not " 0.22 "] for the Nurses' Health Study, 0.29 for the Nurses' Health Study II, and 0.32 [not " 0.28 "] for the Health Professionals Follow-up Study.

In the last paragraph of the results section, the first sentence should have noted that "replacing each three servings/week of fruit juice consumption with the same amount of total or individual whole fruits, the risk of type 2 diabetes in the pooled analysis was 5\% (95\% confidence interval 3\% to 7\%) [not "7\% (95\% confidence interval $4 \%$ to $9 \%$ )"] lower for total whole fruits."

In the figure in the online version, the bar for "Total fruits" should have depicted a hazard ratio and $95 \%$ confidence interval of 0.95 ( 0.93 to 0.97 ) for the "Relative risk of type 2 diabetes."

Lastly, tables 1, 2, and 4 all contain some incorrect values. In table 1, the values for the Nurses' Health Study and the Health Professionals Follow-up Study were incorrect. A corrected version of table 1 is below.

In table 2, the values for the Nurses' Health Study, the Health Professionals Follow-up Study, and the pooled results contained some inaccuracies. A corrected version of table 2 is below.

In table 4 , the hazard ratios and $95 \%$ confidence intervals for the variable of fruit juice should have been different. A corrected version of table 4 is below.

Cite this as: BMJ 2013;347:f6935

๑ BMJ Publishing Group Ltd 2013 


\section{Tables}

Table 1| Baseline characteristics of women in Nurses' Health Study and Nurses' Health Study II and men in Health Professionals Follow-up Study. Values are means (standard deviations) or percentages unless stated otherwise

\begin{tabular}{|c|c|c|c|c|c|}
\hline \multirow[b]{2}{*}{ Characteristics } & \multicolumn{5}{|c|}{ Total whole fruit consumption } \\
\hline & $<4$ servings/week & 4-6 servings/week & 1 serving/day & 2 servings/day & $\geq 3$ servings/day \\
\hline \multicolumn{6}{|l|}{ Nurses' Health Study*: } \\
\hline No of participants & 16069 & 11698 & 15783 & 15106 & 7449 \\
\hline Age (years) & $48.2(6.9)$ & $49.1(7.0)$ & $50.0(7.1)$ & $51.1(7.1)$ & $52.3(6.9)$ \\
\hline Body mass index & $24.7(4.8)$ & $24.8(4.6)$ & $24.9(4.6)$ & $25.0(4.5)$ & $24.9(4.5)$ \\
\hline Physical activity (MET hours/week) & $10.6(18.2)$ & $12.4(17.8)$ & $14.1(19.0)$ & $16.2(21.3)$ & $20.2(27.6)$ \\
\hline Alcohol intake (g/day) & $9.2(14.1)$ & $7.2(11.1)$ & $6.4(10.4)$ & $5.9(9.3)$ & $5.3(9.0)$ \\
\hline Current smoker (\%) & 38 & 27 & 20 & 16 & 14 \\
\hline White (\%) & 98 & 98 & 98 & 98 & 97 \\
\hline Family history of diabetes (\%) & 24 & 26 & 26 & 26 & 26 \\
\hline Multivitamin use (\%) & 31 & 35 & 38 & 41 & 43 \\
\hline Ever menopausal hormone use (\%) & 17 & 19 & 22 & 25 & 28 \\
\hline Total energy intake (kcal/day) & $1566(506)$ & $1672(498)$ & $1741(500)$ & $1857(516)$ & $2041(543)$ \\
\hline Fruit juice consumption (serving/day) & $0.54(0.70)$ & $0.67(0.66)$ & $0.75(0.69)$ & $0.82(0.74)$ & $0.95(0.96)$ \\
\hline Modified alternate healthy eating index score & $44.8(9.9)$ & $47.1(9.8)$ & $49.2(9.9)$ & $51.4(10.0)$ & $54.1(10.1)$ \\
\hline \multicolumn{6}{|l|}{ Nurses' Health Study IIt: } \\
\hline No of participants & 25120 & 16726 & 20259 & 16312 & 6687 \\
\hline Age (years) & $35.9(4.7)$ & $36.0(4.7)$ & $36.1(4.6)$ & $36.3(4.6)$ & $36.4(4.6)$ \\
\hline Body mass index & $24.6(5.6)$ & $24.6(5.3)$ & $24.5(5.0)$ & $24.5(4.9)$ & $24.3(5.0)$ \\
\hline Physical activity (MET hours/week) & $15.8(23.0)$ & $18.7(23.7)$ & $21.3(25.6)$ & $25.7(30.6)$ & $33.2(39.6)$ \\
\hline Alcohol intake (g/day) & $3.6(7.3)$ & $3.0(5.8)$ & $3.0(5.5)$ & $3.0(5.4)$ & $2.8(5.4)$ \\
\hline Current smoker (\%) & 18 & 12 & 9 & 8 & 7 \\
\hline White (\%) & 96 & 96 & 96 & 96 & 94 \\
\hline Family history of diabetes (\%) & 16 & 16 & 16 & 17 & 16 \\
\hline Multivitamin use (\%) & 37 & 42 & 46 & 50 & 52 \\
\hline Ever menopausal hormone use (\%) & 3 & 3 & 3 & 3 & 3 \\
\hline Current oral contraceptive use (\%) & 12 & 12 & 11 & 10 & 9 \\
\hline Total energy intake (kcal/day) & $1559(504)$ & $1714(502)$ & $1820(504)$ & $1983(516)$ & $2215(551)$ \\
\hline Fruit juice consumption (serving/day) & $0.45(0.67)$ & $0.61(0.70)$ & $0.70(0.76)$ & $0.82(0.84)$ & $1.02(1.09)$ \\
\hline Modified alternate health eating index score & $45.3(10.3)$ & $48.0(10.3)$ & $50.1(10.3)$ & $52.4(10.3)$ & $54.8(10.3)$ \\
\hline \multicolumn{6}{|l|}{ Health Professionals Follow-up Studył: } \\
\hline No of participants & 8397 & 5662 & 8193 & 8565 & 5356 \\
\hline Age (years) & $50.1(8.4)$ & $51.1(8.9)$ & $52.2(9.2)$ & $53.7(9.5)$ & $55.0(9.6)$ \\
\hline Body mass index & $25.1(5.0)$ & $25.0(4.7)$ & $25.0(4.6)$ & $24.9(4.7)$ & $24.6(5.0)$ \\
\hline Physical activity (MET hours/week) & $16.2(24.0)$ & $18.0(22.5)$ & $21.3(31.8)$ & $23.9(29.4)$ & $29.7(38.9)$ \\
\hline Alcohol intake (g/day) & $14.7(18.4)$ & $12.0(15.8)$ & $11.0(14.4)$ & $10.0(13.8)$ & $8.4(12.2)$ \\
\hline Current smoker (\%) & 18 & 11 & 8 & 6 & 4 \\
\hline White (\%) & 95 & 95 & 95 & 96 & 95 \\
\hline Family history of diabetes (\%) & 18 & 18 & 19 & 20 & 19 \\
\hline Multivitamin use (\%) & 37 & 39 & 42 & 44 & 47 \\
\hline Total energy intake (kcal/day) & $1814(579)$ & 1915 (592) & $1973(592)$ & $2100(608)$ & $2287(644)$ \\
\hline Fruit juice consumption (serving/day) & $0.57(0.70)$ & $0.69(0.72)$ & $0.78(0.77)$ & $0.87(0.84)$ & $1.01(1.08)$ \\
\hline Modified alternate health eating index score & $48.2(10.8)$ & $51.1(10.7)$ & $53.3(10.5)$ & $55.7(10.5)$ & $58.7(10.4)$ \\
\hline
\end{tabular}

$\mathrm{MET}=$ metabolic equivalent of task. 
Table 1 (continued)

Total whole fruit consumption

*Baseline was 1984

†Baseline was 1991

‡Baseline was 1986 . 
Table 2| Pooled hazard ratios (95\% confidence intervals) of type 2 diabetes for total whole fruit consumption in Nurses' Health Study, Nurses' Health Study II, and Health Professionals Follow-up Study

\begin{tabular}{|c|c|c|c|c|c|c|c|c|}
\hline \multirow[b]{2}{*}{ Variables } & \multicolumn{5}{|c|}{ Consumption levels } & \multirow[b]{2}{*}{ Linear trend* } & \multicolumn{2}{|c|}{$P$ values } \\
\hline & $\begin{array}{c}<4 \\
\text { servings/week }\end{array}$ & $\begin{array}{c}5-6 \\
\text { servings/week }\end{array}$ & 1 serving/day & 2 servings/day & $\geq 3$ servings/day & & For trend & $\begin{array}{c}\text { For } \\
\text { heterogeneity }\end{array}$ \\
\hline $\begin{array}{l}\text { Nurses' Health } \\
\text { Study†: }\end{array}$ & 1380/270 193 & $1158 / 241555$ & $1593 / 359754$ & $1516 / 358670$ & $711 / 163955$ & & & \\
\hline $\begin{array}{l}\text { Adjusted hazard } \\
\text { ratioł }\end{array}$ & 1.00 & 0.92 (0.85 to 0.99$)$ & 0.86 (0.80 to 0.93$)$ & 0.86 (0.79 to 0.93$)$ & $0.90(0.81$ to 0.99$)$ & $\begin{array}{c}0.98(0.96 \text { to } \\
1.00)\end{array}$ & & \\
\hline $\begin{array}{l}\text { Nurses' Health } \\
\text { Study II†: }\end{array}$ & $995 / 358762$ & $620 / 287918$ & $718 / 368180$ & $598 / 295904$ & $222 / 105347$ & & & \\
\hline $\begin{array}{l}\text { Adjusted hazard } \\
\text { ratiof }\end{array}$ & 1.00 & 0.86 (0.77 to 0.95$)$ & 0.84 (0.76 to 0.94$)$ & 0.88 (0.78 to 0.98$)$ & $0.92(0.78$ to 1.08$)$ & $\begin{array}{c}0.99(0.96 \text { to } \\
1.02)\end{array}$ & & \\
\hline $\begin{array}{l}\text { Health } \\
\text { Professionals } \\
\text { Follow-up Studyt: }\end{array}$ & $636 / 134159$ & $454 / 101290$ & $625 / 156230$ & $627 / 164411$ & $345 / 98313$ & & & \\
\hline $\begin{array}{l}\text { Adjusted hazard } \\
\text { ratiof }\end{array}$ & 1.00 & 1.00 (0.88 to 1.12$)$ & $0.92(0.82$ to 1.03$)$ & 0.89 (0.79 to 1.01$)$ & $\begin{array}{c}0.90(0.78 \text { to } \\
1.04)\end{array}$ & $\begin{array}{c}0.98(0.95 \text { to } \\
1.00)\end{array}$ & & \\
\hline Pooled results $\ddagger \S$ & 1.00 & 0.91 (0.86 to 0.97 ) & 0.87 (0.82 to 0.92$)$ & 0.87 (0.82 to 0.92$)$ & 0.90 (0.84 to 0.97$)$ & $\begin{array}{c}0.98 \text { (0.97 to } \\
0.99)\end{array}$ & 0.003 & 0.73 \\
\hline
\end{tabular}

*Estimated based on every three servings/week increment.

†Cases/person years of observation.

¥Adjusted for age (years), ethnicity (white, African-American, Hispanic, or Asian), body mass index (<23, 23.0-24.9, 25.0-26.9, 27.0-28.9, 29.0-30.9, 31.0-32.9, 33.0-34.9, 35.0-36.9, 37.0-38.9, 39.0-40.9, 41.0-42.9, 43.0-44.9, $\geq 45.0$, or missing), smoking status (never, former, current (1-14, 15-24, or $\geq 25$ cigarettes/day), or missing), multivitamin use (yes or no), physical activity (<3, 3.0-8.9, 9.0-17.9, 18.0-26.9, $\geq 27.0$ MET (metabolic equivalent of task) hours/week, or missing), family history of diabetes (yes or no), menopausal status and post-menopausal hormone use (premenopause, post-menopause (never, former, or current hormone use), or missing, for women), oral contraceptive use (yes, no, or missing, for Nurses' Health Study II) total energy intake (kcal/day), fruit juice consumption (<1, 1, 2-4, 5-6, $\geq 7$ servings/week) and the modified alternate healthy eating index score (fifths). Individual fruit consumption was mutually adjusted. §Study estimates from three cohorts were pooled using a fixed effects model. 
Table 4| Pooled hazard ratios (95\% confidence intervals) of type 2 diabetes* for consumption of fruit juice and fruits grouped by their glycemic index/glycemic load in Nurses' Health Study, Nurses' Health Study II, and Health Professionals Follow-up Study

\begin{tabular}{|c|c|c|c|c|c|c|c|c|}
\hline \multirow[t]{2}{*}{ Variables } & \multicolumn{5}{|c|}{ Consumption levels } & \multirow[t]{2}{*}{ Linear trend $\dagger$} & \multicolumn{2}{|c|}{$P$ value } \\
\hline & $\begin{array}{c}<1 \\
\text { serving/week }\end{array}$ & 1 serving/week & $\begin{array}{c}2-4 \\
\text { servings/week }\end{array}$ & $\begin{array}{c}5-6 \\
\text { servings/week }\end{array}$ & $\geq 1$ serving/day & & For trend & $\begin{array}{c}\text { For } \\
\text { heterogeneity }\end{array}$ \\
\hline \multicolumn{9}{|c|}{ Glycemic loadł: } \\
\hline High & 1.00 & 0.89 (0.81 to 0.98$)$ & $0.89(0.82$ to 0.96$)$ & 0.81 (0.74 to 0.88$)$ & 0.83 (0.76 to 0.91$)$ & $\begin{array}{c}0.93 \text { (0.91 to } \\
0.96)\end{array}$ & $<0.001$ & 0.13 \\
\hline Moderate & 1.00 & 0.96 (0.90 to 1.03$)$ & $0.96(0.90$ to 1.02$)$ & $0.97(0.90$ to 1.05$)$ & 1.03 (0.94 to 1.13$)$ & $\begin{array}{c}1.01 \text { (0.98 to } \\
1.04)\end{array}$ & 0.61 & 0.85 \\
\hline Low & 1.00 & $0.94(0.89$ to 1.01$)$ & $0.96(0.90$ to 1.01$)$ & $0.92(0.86$ to 1.00$)$ & 0.93 (0.86 to 1.02$)$ & $\begin{array}{c}0.98(0.94 \text { to } \\
1.01)\end{array}$ & 0.14 & 0.04 \\
\hline \multicolumn{9}{|l|}{$\begin{array}{l}\text { Glycemic } \\
\text { index§: }\end{array}$} \\
\hline High & 1.00 & $0.99(0.92$ to 1.07$)$ & 0.94 (0.88 to 1.01$)$ & 0.92 (0.85 to 0.99) & 0.99 (0.91 to 1.07$)$ & $\begin{array}{c}0.98 \text { (0.95 to } \\
1.01)\end{array}$ & 0.21 & 0.20 \\
\hline Moderate & 1.00 & $0.96(0.91$ to 1.01$)$ & $0.89(0.84$ to 0.94$)$ & $0.87(0.80$ to 0.94$)$ & 0.94 (0.85 to 1.05$)$ & $\begin{array}{c}0.94 \text { (0.90 to } \\
0.97)\end{array}$ & $<0.001$ & 0.97 \\
\hline Low & 1.00 & $0.90(0.82$ to 1.00$)$ & $0.87(0.80$ to 0.94$)$ & 0.85 (0.78 to 0.93$)$ & 0.87 (0.80 to 0.95$)$ & $\begin{array}{c}0.97 \text { (0.94 to } \\
1.00)\end{array}$ & 0.06 & 0.58 \\
\hline Fruit juiceף & 1.00 & 1.03 (0.95 to 1.10$)$ & 1.04 (0.98 to 1.11$)$ & $1.06(1.00$ to 1.13$)$ & $1.17(1.11$ to 1.24$)$ & $\begin{array}{c}1.07 \text { (1.04 to } \\
1.09)\end{array}$ & $<0.001$ & 0.84 \\
\hline
\end{tabular}

*Adjusted for age (years), ethnicity (white, African American, Hispanic, or Asian), body mass index (<23, 23.0-24.9, 25.0-26.9, 27.0-28.9, 29.0-30.9, 31.0-32.9, 33.0-34.9, 35.0-36.9, 37.0-38.9, 39.0-40.9, 41.0-42.9, 43.0-44.9, $\geq 45.0$, or missing), smoking status (never, former, current (1-14, 15-24, or $\geq 25$ cigarettes/day), or missing), multivitamin use (yes or no), physical activity (<3, 3.0-8.9, 9.0-17.9, 18.0-26.9, $\geq 27.0 \mathrm{MET}$ (metabolic equivalent of task) hours/week, or missing), family history of diabetes (yes or no), menopausal status and post-menopausal hormone use (premenopause, post-menopause (never, former, or current hormone use), or missing, for women), oral contraceptive use (yes, no, or missing, for Nurses' Health Study II) total energy intake (kcal/day), and modified alternate healthy eating index score (fifths). Results were pooled using a fixed effects model.

†Linear trend was modeled based on every three servings/week increment.

‡High glycemic load fruits included prunes, bananas, grapes, raisins, apples, and pears (glycemic load per serving 8.1-19.2), moderate glycemic load fruits included cantaloupe, oranges, and blueberries (5.7-8.0), and low glycemic load fruits included peaches, plums, apricots, grapefruit, and strawberries (1.3-5.6). Fruit juice consumption (<1, 1, 2-4, or 5-6 servings/week, or $\geq 1$ serving/day) was further adjusted, and consumption of high, moderate, and low glycemic load fruits was mutually adjusted.

$\S$ High glycemic index fruits included cantaloupe, bananas, grapes, raisins (glycemic index 60-70), moderate glycemic index fruits included prunes, blueberries, and grapefruit (47-59), and low glycemic index fruits included apples, pears, oranges, peaches, plums, apricots, and strawberries (34-46). Fruit juice consumption $(<1,1,2-4$, or 5-6 servings/week, or $\geq 1$ serving/day) was further adjusted, and consumption of high, moderate, and low glycemic index fruits was mutually adjusted. IFruit juice included apple, orange, grapefruit, and other. Total whole fruit consumption ( $<4$, or 4-6 servings/week, or 1,2 , or $\geq 3$ servings/day) was further adjusted. 